\title{
Aspergilose broncopulmonar alérgica em pacientes com Fibrose Cística
}

\author{
Allergic bronchopulmonary aspergillosis in patients with Cystic Fibrosis \\ Aspergilosis broncopulmonar alérgica em pacientes com Fibrosis Quística
}

Recebido: 13/06/2021 | Revisado: 17/06/2021 | Aceito: 17/06/2021 | Publicado: 18/06/2021

\author{
Reinaldo José dos Santos Júnior \\ ORCID: https://orcid.org/0000-0003-1208-6975 \\ Universidade do Vale do Ipojuca, Brasil \\ E-mail: reinaldosantosjunior123456@gmail.com \\ Natália Milena Silva \\ ORCID: https://orcid.org/0000-0003-0356-2343 \\ Universidade do Vale do Ipojuca, Brasil \\ E-mail: nataliamillena18@outlook.com \\ Pâmella Grasielle Vital Dias de Souza \\ ORCID: https://orcid.org/0000-0001-9884-9544 \\ Universidade do Vale do Ipojuca, Brasil \\ E-mail: pamellagvds@gmail.com
}

\section{Resumo}

A fibrose cística (FC) é uma doença genética de perfil autossômico recessivo e é caracterizada pela disfunção do gene CFTR, o que irá afetar alguns órgãos como pulmão, pâncreas, fígado, intestino e testículos, sendo os pulmões o mais acometido. Tais alterações são capazes de provocar obstruções consistentes devido ao muco viscoso produzido. As infecções são um fator recorrente nos portadores dessa doença devido a presença da secreção, tornando-se um ambiente ideal para propagação de micro-organismos, como fungos e bactérias, que podem colonizar as vias aéreas dos pacientes e provocar agravos, piorando o quadro clínico do paciente. Como é o caso do fungo Aspergillus fumigattus, causador da aspergilose broncopulmonar alérgica (ABPA), que pode acarretar relevantes complicações principalmente nas funções pulmonares. Analisando os fatores que faz com que os pacientes com FC tenha maior facilidade em contrair a ABPA e como isso pode agravar seu quadro clínico, com busca de materiais a partir das plataformas PUBMED e SCIELO, utilizando as recomendações do Preferred Reporting Items for Systematic Reviews and Meta-Analyses (PRISMA), os resultados obtidos nesse estudo mostram que a presença de ABPA nos fibrocísticos favorecem um aumento nas colonizações microbianas, deterioração pulmonar, pior estado nutricional além de maior obstrução respiratória por muco, contribuindo pelo aumento nos índices de hospitalização.

Palavras-chave: Fibrose cística; Aspergillus fumigastus; Aspergilose broncopulmonar alérgica.

\begin{abstract}
Cystic fibrosis (CF) is a genetic disease of autosomal recessive profile and is characterized by dysfunction of the CFTR gene, which will affect some organs such as the lungs, pancreas, liver, intestines, and testes, with the lungs being the most characteristic. Such changes are capable of causing consistent obstructions due to the viscous mucus produced. Infections are a recurrent factor in carriers of this disease due to the presence of secretion, becoming an ideal environment for the propagation of microorganisms, such as fungi and bacteria, which can colonize the airways of patients and bring about an aggravation, worsening the patient's clinical picture. This is the case of the fungus Aspergillus fumigattus, the cause of allergic bronchopulmonary aspergillosis (ABPA), which can lead to relevant complications, especially in lung function. Analyzing the factors that make $\mathrm{CF}$ patients more likely to contract ABPA and how this can worsen their clinical condition, searching for materials from the PUBMED and SCIELO platforms, using the recommendations of the Preferred Reporting Items for Systematic Reviews and Meta-Analyses (PRISMA), the results obtained in this study show that the presence of ABPA in cystic fibrosis patients brings increased microbial colonization, lung deterioration, poorer nutritional status, and greater respiratory obstruction by mucus that causes a greater increase in hospitalization.
\end{abstract}

Keywords: Cystic fibrosis; Aspergillus fumigatus; Allergic bronchopulmonary aspergillosis.

\section{Resumen}

La fibrosis quística (FQ) es una enfermedad genética de perfil autosómico recesivo y se caracteriza por la disfunción del gen CFTR, que afectará a algunos órganos como los pulmones, el páncreas, el hígado, el intestino y los testículos, siendo los pulmones los más característicos. Estas alteraciones son capaces de provocar obstrucciones consistentes debido al moco viscoso producido. Las infecciones son un factor recurrente en los portadores de esta enfermedad debido a la presencia de secreciones, convirtiéndose en un ambiente ideal para la propagación de microorganismos como hongos y bacterias, que pueden colonizar las vías respiratorias de los pacientes y traer un agravamiento, empeorando el cuadro clínico del paciente. Es el caso del hongo Aspergillus fumigattus, causante de la aspergilosis broncopulmonar alérgica (ABPA), que puede dar lugar a complicaciones relevantes, especialmente en la función pulmonar. Analizando los factores 
que hacen que los pacientes con FQ sean más propensos a contraer ABPA y cómo esto puede empeorar su condición clínica, con una búsqueda de materiales de las plataformas PUBMED y SCIELO, utilizando las recomendaciones de los Preferred Reporting Items for Systematic Reviews and Meta-Analyses (PRISMA), los resultados obtenidos en este estudio muestran que la presencia de ABPA en los pacientes con fibrosis quística conlleva un aumento de la colonización microbiana, deterioro pulmonar, peor estado nutricional y mayor obstrucción respiratoria por mucosidad, lo que provoca un mayor aumento de la hospitalización.

Palabras clave: Fibrosis quística; Aspergillus fumigatus; Aspergilosis broncopulmonar alérgica.

\section{Introdução}

A fibrose cística é uma doença genética causada a partir de uma mutação no cromossomo 7, esta mutação, segundo a literatura, é a responsável pelo comprometimento no sistema respiratório (SR), aparelho reprodutor masculino e glândulas exócrinas. Este comprometimento do SR, faz com que haja uma produção de muco espesso e viscoso nos pulmões, tornando um microambiente ideal para o desenvolvimento de micro-organismos, possibilitando uma maior facilidade dos pacientes em contrair infecções que afeta o comprometimento pulmonar, visto que, o acometimento pulmonar é atualmente o maior fator de morbidade, e as complicações acometida no sistema respiratório acaba sendo a principal causa de mortalidade em pacientes fibrocísticos (Bonfim et al., 2019).

Os agentes bacterianos mais vistos nesses casos são Staphylococcus aureus, Haemophius influenzae, Pseudomonas aeruginosa e Burkhoderia cepaci. Já os agentes fúngicos é comum a presença da espécie Aspergillus spp. sendo mais recorrente o Aspergillus fumigatus (Hoffmann; Procianoy, 2011). Essas infecções do trato respiratório costumam acontecer logo após o nascimento de criança com FC e podem acompanhar de respostas inflamatórias intensas que podem prejudicar ainda mais o quadro clínico desses pacientes, como o caso da aspergilose broncopulmonar alérgica (ABPA), doença que acomete principalmente pacientes com asma ou fibrose cística. Em geral, esses pacientes desenvolvem um quadro de hipersensibilidade a antígenos do Aspergillus fumigatus, que pode desencadear obstruções nas vias respiratórias causando bronquiectasia e fibrose pulmonar, que acaba trazendo comprometimento pulmonar, podendo ser fatal nesses pacientes (Firmida et al., 2011).

Esse estudo apresenta importantância, visto que, a susceptibilidade infeciosa nesses pacientes é de extrema relevância, onde esses indivíduos necessitam de maiores precauções diante de infecções microbianas, visando uma maior sobrevida e qualidade de vida dos mesmos, onde o reconhecimento e tratamento precoce das infecções fúngica são essenciais para prevenir a progressão da FC (Castro; Firmida, 2011). Os estudos acerca desse tema necessitam ser mais proeminente, entretanto, é notório que há um declínio na qualidade respiratória desses pacientes devido ao aumento da deterioração pulmonar, e consequentemente ocorrendo um aumento de necessidade hospitalar com a presença da ABPA. Por tanto, este estudo teve como objetivo analisar os fatores predisponentes que os pacientes com FC tem de contrair a ABPA, doença causada pelo fungo Aspergillus fumigatus, e como essa resposta de hipersensibilidade pode trazer malefícios a saúde do paciente

\section{Metodologia}

\section{Métodos}

Esse estudo trata-se de uma revisão de literatura sistêmica e de cunho qualitativa, empregando os critérios e instruções do Preferred Reporting Items for Systematic Reviews and Meta-Analyses (PRISMA) para a pesquisa dos artigos, com o objetivo final de obtenção de dados para o desenvolvimento desta pesquisa (Moher D et al., 2015). 


\section{Critérios de elegibilidade}

Para a obtenção de artigos para análise e leitura foram adicionados nesse estudo artigos, revistas e livros que foram publicados entre o período de 2000 a 2020, preferencialmente da língua portuguesa ou inglesa nos quais tivesse relação com o tema abordado. Sendo assim excluso os trabalhos que não cumpriram os requisitos, os que possuem materiais duplicados, que não utilizam os descritores selecionados ou que estivessem disponíveis de forma incompleta.

\section{Estratégia de busca bibliográfica}

As referências bibliográficas utilizado nesse estudo coletadas nas bases de dados das plataformas PUBMED e SCIELO, que estava de acordo com os critérios de elegibilidade, aplicando como descritores "Fibrose Cística", "Aspergillus fumigatus" e "Aspergilose broncopulmonar alérgica", as quais estão descritas na plataforma de Descritores em Ciências da Saúde (DeCS).

\section{Seleção dos estudos}

Foi feito a seleção de pesquisa a partir da leitura de título e resumos que tem consonância com o tema delimitado e assim, descartando os artigos que não atendiam aos critérios de inclusão nas bases de dados, foi realizado a leitura das publicações e a separação de acordo com a maior relevância entre os assuntos, e por último, o aproveitamento dos artigos selecionados para a formação e compilação dos dados. A Figura 1, retrata as etapas seguidas para a construção deste estudo.

Figura 1 - Etapas seguidas para a construção do estudo.
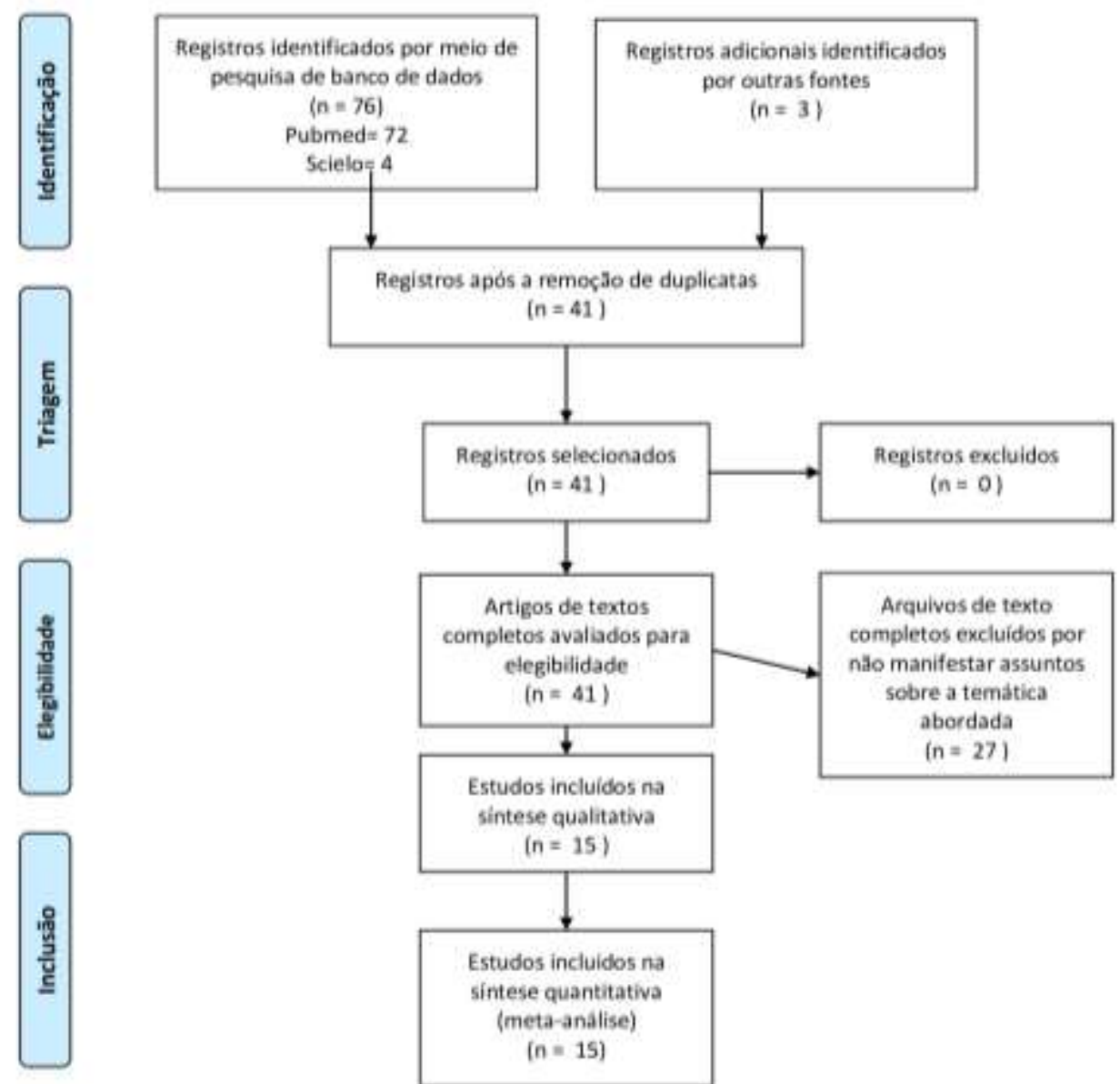

Fonte: Autores. 


\section{Resultados e Discussão}

Este estudo trata-se de uma revisão sistêmica realizada conforme o modelo Preferred Reporting Items for Systematic Reviews and Meta-Analyses (PRISMA), foram encontrados 76 artigos utilizando os descritores: "Fibrose cística”, “Aspergillus fumigatus" e "Aspergilose broncopulmonar alérgica”, sendo selecionado os estudos que manifestaram melhores relações com o tema abordado. De acordo com os critérios de inclusão e exclusão descritos no estudo, o quadro 1 mostra os resultados obtidos:

Quadro 1: Apresentação da síntese de artigos incluídos na revisão

\begin{tabular}{|c|c|c|c|c|}
\hline TÍTULO & AUTOR/ANO & INTERVENÇÃO ESTUDADA & RESULTADOS & $\begin{array}{c}\text { RECOMENDAÇÕES/ } \\
\text { CONCLUSÃO }\end{array}$ \\
\hline $\begin{array}{l}\text { Prevalence of allergic } \\
\text { bronchopulmonary } \\
\text { aspergillosis in cystic } \\
\text { fibrosis patients using } \\
\text { two different } \\
\text { diagnostic criteria }\end{array}$ & $\begin{array}{l}\text { M. Maleki et al, } \\
2020\end{array}$ & $\begin{array}{l}\text { Os autores avaliaram a } \\
\text { prevalência de ABPA em } \\
\text { pacientes iranianos com FC por } \\
\text { dois critérios diagnósticos da } \\
\text { ISHAM e da CF Foundation } \\
\text { Consensus Conference. }\end{array}$ & $\begin{array}{l}88,9 \% \text { dos pacientes com FC com } \\
\text { ABPA e } 74,2 \% \text { dos pacientes com } \\
\text { FC sem ABPA foram positivos } \\
\text { para Aspergillus em amostras de } \\
\text { escarro, e também foi sugerida } \\
\text { que valores de IgE maiores que } \\
1000 \text { UI / mL podem ser um fator } \\
\text { importante na previsão de ABPA. }\end{array}$ & $\begin{array}{l}\text { No devido estudo feitos com } \\
\text { pacientes com FC, } 10,5 \% \text { deles } \\
\text { foram diagnosticados com } \\
\text { ABPA, e todos pacientes com } \\
\text { ABPA mostraram confirmação } \\
\text { de bronquiectasias em } \\
\text { identificados na TCAR. }\end{array}$ \\
\hline $\begin{array}{l}\text { Allergic } \\
\text { Bronchopulmonary } \\
\text { Aspergillosis }\end{array}$ & R. Agarwal, 2009 & $\begin{array}{l}\text { O autor desse estudo, mostra a } \\
\text { aspergilose broncopulmonar } \\
\text { alérgica de modo geral, tais } \\
\text { como história natural da doença, } \\
\text { diagnóstico, epidemiologia, } \\
\text { patogenicidade, complicações e } \\
\text { terapias. }\end{array}$ & $\begin{array}{l}\text { As respostas imunológicas dos } \\
\text { pacientes com ABPA são } \\
\text { importantes para a patogênese e } \\
\text { pode determinar as características } \\
\text { clínicas, biológicas, patológicas e } \\
\text { radiológicas dessa doença. }\end{array}$ & $\begin{array}{l}\text { Importância de seguir os } \\
\text { critérios de diagnóstico de } \\
\text { ABPA para um prognóstico } \\
\text { mais preciso. }\end{array}$ \\
\hline $\begin{array}{l}\text { Aspergillus Infections } \\
\text { and Progression of } \\
\text { Structural Lung } \\
\text { Disease in Children } \\
\text { with Cystic Fibrosis }\end{array}$ & $\begin{array}{l}\text { O. Breuer et al, } \\
2019\end{array}$ & \begin{tabular}{|l|} 
Os autores avaliaram as \\
as sociações longitudinais entre \\
as infecções de Aspergillus e \\
doenças pulmonares em crianças \\
comFC
\end{tabular} & $\begin{array}{l}\text { Foi notada uma condição } \\
\text { respiratória inferior nos pacientes } \\
\text { com FC acometidos com ABPA, } \\
\text { que está as sociada a progressão } \\
\text { da doença pulmonar nas crianças } \\
\text { estudadas. }\end{array}$ & $\begin{array}{l}\text { Ressalta a importância da } \\
\text { triagem de rotina das infecções } \\
\text { de Aspergillus visando evitar a } \\
\text { infecções nos pacientes, } \\
\text { vis ando uma melhor qualidade } \\
\text { respiratória. }\end{array}$ \\
\hline $\begin{array}{l}\text { Risk factors for } \\
\text { persistent Aspergillus } \\
\text { respiratory isolation } \\
\text { in cystic fibrosis }\end{array}$ & G. Hong et al, 2018 & $\begin{array}{l}\text { Os autores observaram a } \\
\text { correlação entre o } \\
\text { desenvolvimento de Aspergillus } \\
\text { no isolamento respiratório e as } \\
\text { características clínicas de cada } \\
\text { paciente. }\end{array}$ & $\begin{array}{l}\text { O Aspergillus fumigatus está } \\
\text { relacionado a uma piora mais } \\
\text { acelerada da função pulmonar e } \\
\text { causando aumento na } \\
\text { mortalidade, e o uso de } \\
\text { antibióticos inalados e orais } \\
\text { podem aumentar os riscos de } \\
\text { contrair a Aspergillus fumigatus. }\end{array}$ & $\begin{array}{l}\text { o uso de macrolídeos e não } \\
\text { macrolídeos, além de } \\
\text { corticosteroides inalatórios } \\
\text { estão contribuindo para maior } \\
\text { risco de infecções a } \\
\text { Aspergillus fumigatus. }\end{array}$ \\
\hline $\begin{array}{l}\text { Influence of } \\
\text { Interleukin- } 10 \text { on } \\
\text { Aspergillus fumigatus } \\
\text { Infection in Patients } \\
\text { with Cystic Fibrosis }\end{array}$ & $\begin{array}{l}\text { J. Brouard et al, } \\
2005\end{array}$ & $\begin{array}{l}\text { O estudo foi elaborado para } \\
\text { investigar uma pos sível } \\
\text { as sociação entre alelos } \\
\text { transportados na posição } 1082 \\
\text { na região promotora do IL-10 } \\
\text { gene e dados clínicos pacientes } \\
\text { com FC. }\end{array}$ & $\begin{array}{l}\text { Os autores sugerem que } \\
\text { polimorfismos na região } \\
\text { promotora de IL-10 pode } \\
\text { influenciar na maneira os } \\
\text { pacientes com FC respondem a } \\
\text { infecção de A. fumigatus. }\end{array}$ & $\begin{array}{l}\text { Foi visto que esses } \\
\text { polimorfis mos na região de IL- } \\
10 \text { aumentamo risco dos } \\
\text { pacientes desenvolverem } \\
\text { infecção crônica ou ABPA. }\end{array}$ \\
\hline \begin{tabular}{|l|} 
Prevalência de \\
aspergilose \\
broncopulmonar \\
alérgica em pacientes \\
com fibrose na Bahia, \\
Brasil \\
\end{tabular} & C. Ana et al, 2008 & $\begin{array}{l}\text { Os autores deste trabalho } \\
\text { analis aram a prevalência da } \\
\text { ABPA em pacientes com FC em } \\
\text { um grupo de pacientes na Bahia. }\end{array}$ & $\begin{array}{l}\text { A prevalência de ABPA foi de } \\
2,7 \% \text { no grupo fibrocístico } \\
\text { estudado. }\end{array}$ & $\begin{array}{l}\text { O nível alto de IgE total nos } \\
\text { pacientes com FC é indicativo } \\
\text { de ABPA, e os indivíduos que } \\
\text { apresentam devem ser } \\
\text { acompanhado com precaução }\end{array}$ \\
\hline
\end{tabular}

Fonte: Autores.

Segundo a pesquisa de Maleki (2020), analisando 86 pacientes portadores de FC de diferentes localidades que estavam internado no hospital Masih Daneshvari no Irã, foi utilizando diversas técnicas de diagnóstico para ABPA, como o teste de função pulmonar, teste de IgE e IgG para Aspergillus, contagem de eosinófilos no sangue periférico, tomografia computadorizada de alta definição (TCAR) e processamento de expectoração. E de acordo com os testes utilizados, a 
prevalência de ABPA nesse grupo de pacientes, foi de 9 indivíduos (10,5\%) diagnosticados com ABPA, e todos os pacientes com ABPA mostraram evidências de bronquiectasias identificados na TCAR.

Em pacientes acometidos com ABPA ocorre uma resposta imune de hipersensibilidade do tipo III, devido as respostas clínicas contra os antígenos do fungo Aspergillus fumigatus, ocorrendo assim uma resposta de inflamação alérgica que é mediada por IgE. Com a produção de IgE e o aumento da liberação dos antígenos do Aspergillus fumigatus, inicia-se a ativação de uma resposta imune, mediada por Th2, o que faz com que os níveis do IgE total e TgE específica contra o fungo, seja produzida. Além disso, com a intensificação das respostas Th2, consequentemente ocorre a produção $\operatorname{IgG}$ e $\operatorname{IgA}$ contra o Aspergillus fumigatus. Essas reações de hipersensibilidade mediadas por IgE, podem se desenvolver gradativamente com o aumento da exposição ao alérgeno, podendo assim evoluir com o tempo, onde essa sensibilização acaba aumentando a progressão da doença, como bronquiectasias, infiltrados pulmonares e doenças obstrutiva pulmonar. (Carneiro et al., 2008).

De acordo com Agarwal (2009), a presença de obstrução na mucosa, bronquiectasia e infiltrados pulmonares serem manifestações de ABPA, tanto como também essas serem manifestações comumente nos fibrocísticos, faz ressaltar a importância da utilização dos critérios estabelecidos para o diagnóstico de ABPA. Ainda assim, a presença da aspergilose broncopulmonar alérgica associada a FC irá trazer alguns prejuízos para os mesmos, tais como um aumento nas taxas de colonização microbiana, pneumotórax, hemoptise, deterioração da função pulmonar, acarretando um pior estado nutricional.

Analisando dados longitudinais de 330 crianças do Princess Margaret Hospital e Royal Children's Hospital na Austrália, dentre esse grupo de criança, 35\% apresentaram cultura positiva para Aspergillus. Nesse estudo foi observado que a infecção por Aspergillus aumentaram o risco de presença de doença pulmonar estrutural caracterizada principalmente por obstrução de muco e aprisionamento de ar, além de um aumento no risco de hospitalização (Brever et al., 2019).

A partir de estudos de Hong (2018) utilizando estudo de dados do Cystic Fibrosis Foundation Patient Registry (CFFPR), foi observado que a insuficiência pancreática está associada a maiores chances do desenvolvimento e persistência de Aspergillus, além do uso de antibióticos nos pacientes com FC tanto os fármacos inalados como os orais, tendo o exemplo dos macrolídeos, que podem inibir a infiltração de neutrófilos no trato respiratório, podem trazer uma contribuição para uma alteração do microambiente das vias respiratórias, possibilitando uma maior facilidade para o surgimento dos fungos filamentosos, Aspergillus fumigatus. Porém os achados deste determinado estudo não acredita que a interrupção desses medicamentos como: corticosteroides e antibióticos inalatórios seja benéfica para os pacientes que os necessitam.

Visto que há muito interesse no papel que a interleucina (IL)-10 efetua nos processos pulmonares inflamatórios e infeciosos que são alterados na FC, um estudo feito por Brouard et al (2005) utilizados dados de 378 pacientes dos principais centros de fibrose cística na França, foi notado que a interleucina 10 (IL-10) que é um grande responsável pela regulação de resposta a infecções endo brônquicas e também da resposta imune do Aspergillus fumigatus. foi visto que o genótipo 1082GG do gene da IL-10 está associado como a maior ocorrência de colonização de A. fumigatus e ABPA nos pacientes fibrocísticos, além de que o estudo aponta que os polimorfismosna IL-10 pode influenciar na forma com que os fibrocísticos respondem a infecções do Aspergillus fumigatus desenvolvendo infecções croninca ou ABPA.

\section{Conclusão}

Visto os resultados do presente estudo, pode-se concluir que a presença de aspergilose broncopulmonar alérgica derivada do fungo Aspergillus fumigatus nos pacientes com fibrose cística acarreta, proeminentemente, em uma maior deterioração pulmonar, ocasionada em uma maior produção de muco derivando em maior risco de hospitalização, e de mais riscos de infecções de microorganismos.

Mais estudos devem ser feitos a cerca dessa temática, porem a dificuldade no diagnóstico de ABPA devido a recorrência nas infecções desses pacientes e a falta de uma padronização universal acerca dos achados clínicos, prejudicam os 
estudos acerca desse tema. Para trabalhos futuros sugiro que acompanhe a progressão hospitalar desses pacientes, e a qualidade de vida dos mesmos após essas infecções.

\section{Referências}

Aguiar, S. S., Damaceno, N. \& Forte, W. C. N. (2017). Tests to assess sensitization to Aspergillus fumigatus in cystic fibrosis. Revista Paulista de Pediatria, 35(3), 252-257. https://doi.org/10.1590/1984.0462/;2017;35;3;00003

Agarwal, R. (2012). Aspergilose broncopulmonar alérgica - tratamento. American Review Of Respiratory Disease, 2-3.

Almeida, M. B., Bussamra, M. H. C. F. \& Rodrigues, J. C. (2006). ABPA diagnosis in cystic fibrosis patients: The clinical utility of IgE specific to recombinant Aspergillus fumigatus allergens. Jornal de Pediatria, 82(3), 215-220. https://doi.org/10.2223/JPED.1479

Alyasin, S., Moghtaderi, M., Farjadian, S., Babaei, M. \& Hosseini Teshnizi, S. (2018). Allergic bronchopulmonary aspergillosis in patients with cystic fibrosis and non-cystic fibrosis bronchiectasis. Electronic Physician, 10(1), 6273-6278. https://doi.org/10.19082/6273

Breuer, O., Schultz, A., Garratt, L. W., Turkovic, L., Rosenow, T., Murray, C. P., Karpievitch, Y. V., Akesson, L., Dalton, S., Sly, P. D., Ranganathan, S., Stick, S. M. \& Caudri, D. (2020). Aspergillus infections and progression of structural lung disease in children with cystic fibrosis. American Journal of Respiratory and Critical Care Medicine, 201(6), 688-696. https://doi.org/10.1164/rccm.201908-1585OC

Carneiro, A. C. C., Lemos, A. C. M., Arruda, S. M. \& Santana, M. A. P. S. (2008). Prevalência de aspergilose broncopulmonar alérgica em pacientes com fibrose cística na Bahia, Brasil. Jornal Brasileiro de Pneumologia, 34(11), 900-906. https://doi.org/10.1590/S1806-37132008001100003

César, M. \& Castro, S. De. (2011). O Tratamento na Fibrose Cística e suas Complicações. Revista Hospital Universitário Pedro Ernesto, 10(4). https://doi.org/10.12957/rhupe.2011.8882v

Firmida, M. D. C. (2011). Fisiopatologia e Manifestações Clínicas da Fibrose Cística. Revista Hospital Universitário Pedro Ernesto, 10(4). https://doi.org/10.12957/rhupe.2011.8878

Hong, G., Psoter, K. J., Jennings, M. T., Merlo, C. A., Boyle, M. P., Hadjiliadis, D., Kawut, S. M. \& Lechtzin, N. (2018). Risk factors for persistent Aspergillus respiratory isolation in cystic fibrosis. Journal of Cystic Fibrosis, 17(5), 624-630. https://doi.org/10.1016/j.jcf.2018.01.008

Hoffmann, A. \& Procianoy, E. da F. A. (2011). Infecção respiratória na fibrose cística e tratamento. Clinical and Biomedical Research, 31(2).

Itens, P., Revis, R. \& Uma, P. (2015). Principais itens para relatar Revisões sistemáticas e Meta-análises: A recomendação PRISMA. Epidemiologia e Serviços de Saúde, 24(2), 335-342. https://doi.org/10.5123/s1679-49742015000200017

Lattanzi, C., Messina, G., Fainardi, V., Tripodi, M. C., Pisi, G. \& Esposito, S. (2020). Allergic bronchopulmonary aspergillosis in children with cystic fibrosis: An update on the newest diagnostic tools and therapeutic approaches. Pathogens, 9(9), 1-13. https://doi.org/10.3390/pathogens9090716

Marmitt, D. J., Rempel, C., Goettert, M. I. \& Silva, A. C. (2015). Plantas Medicinais da RENISUS Com Potencial Anti-inflamatório: Revisão Sistemática Em Três Bases de Dados Científicas. Revista Fitos, 9(2). https://doi.org/10.5935/2446-4775.20150011

Maleki, M., Mortezaee, V., Hassanzad, M., Mahdaviani, S. A., Poorabdollah, M., Mehrian, P., Behnampour, N., Mirenayat, M. S., Abastabar, M., Tavakoli, M. \& Hedayati, M. T. (2020). Prevalence of allergic bronchopulmonary aspergillosis in cystic fibrosis patients using two different diagnostic criteria. European Annals of Allergy and Clinical Immunology, 52(3), 104-111. https://doi.org/10.23822/EurAnnACI.1764-1489.121

Sancho-García, I. \& Piñero-Zapata, M. (2021). Aumento de eventos cardiovasculares en pacientes tratados con clopidogrel y omeprazol simultáneamente: una revisión sistemática. Evidentia, 18, e12814. https://doi.org/10.1371/journal.pmed1000097 